\title{
Temporal niche partitioning between carnivores differs depending on the analytical method: comparison between temporal overlap and co- occurrence, and time-to-encounter analyses
}

\section{Ryoga Watabe}

Yamagata University

Hiroshi Tsunoda

Center for Environmental Science in Saitama

Masayuki U. Saito ( $\sim$ saito.ume@gmail.com )

Yamagata University

\section{Research Article}

Keywords: Temporal niche partitioning, carnivores, analytical method, temporal overlap, time-to-encounter analyses, co-occurrence

Posted Date: August 26th, 2021

DOI: https://doi.org/10.21203/rs.3.rs-841897/v1

License: (c) (1) This work is licensed under a Creative Commons Attribution 4.0 International License.

Read Full License 


\section{Abstract}

Temporal niche partitioning is one of the most important strategies for carnivore coexistence. Cameratrap data has been analyzed through several methods to assess the temporal niche partitioning. When different analytical approaches used to evaluate niche partitioning detect different results, the mechanisms of species coexistence may be misinterpreted. As such, it is important to evaluate the temporal niche partitioning from multiple analytical approaches. In this study, we compared three analytical methods-the temporal overlap, temporal co-occurrence, and time-to-encounter analysis-to evaluate the temporal niche partitioning among sympatric medium-sized carnivores: red foxes, raccoon dogs, and Japanese martens. From May to October 2019 and 2020, we obtained the activity of the target species using camera-traps in northeastern Japan. We analyzed the data with the coefficient of temporal overlap, probabilistic co-occurrence analysis, checkerboard score, and multi-response permutation procedures. The results of the assessment of the niche partitioning differed depending on the analytical methods. Therefore, we conclude that the choice of analytical approach is important for understanding the mechanisms of species coexistence through temporal niche partitioning.

\section{Introduction}

The competitive exclusion principle states that two ecologically similar species cannot coexist ${ }^{1}$. Thus, multiple sympatric species can partition their niche according to four major factors: food resources, natural enemies, space, and time ${ }^{2}$. Elucidating the mechanisms of species coexistence based on their niche partitioning is important for understanding community diversity and for implementing effective community conservation and management strategies ${ }^{3-6}$.

It is widely known that interspecific competition can often occur among sympatric carnivores ${ }^{7-10}$. The extent of their interspecific competition is influenced by taxonomic similarity, dietary overlap, and intermediate body-size differences ${ }^{11}$. For example, larger coyotes (Canis latrans) can exclude smaller swift foxes (Vulpes velox) from their home ranges and territories ${ }^{9}$. Similarly, Tsunoda et al. ${ }^{12}$ suggested that interspecific competition can increase between larger golden jackals (Canis aureus) and smaller red foxes (Vulpes vulpes) because of their dietary overlaps. To avoid such competition, carnivores coexist sympatrically by shifting their activity time and spaces (e.g., ${ }^{13-15}$ ). In particular, temporal niche partitioning is one of the most important strategies to ensure their coexistence $e^{5,16,17}$.

Currently, temporal niche partitioning among sympatric carnivores is often assessed using camera-trap data $^{5}$, and several studies have demonstrated that temporal niche partitioning is key for their successful sympatry (e.g., ${ }^{18-21}$ ). For example, European badgers (Meles meles) and stone martens (Martes foina) shifted their diel activity patterns to avoid antagonistic encounters with larger golden jackals in Bulgaria $^{20}$. Stone martens also tended to be active at different times than larger red foxes and European wildcats (Felis sylvestris) ${ }^{21}$. Typically, temporal niche partitioning among carnivores is assessed using time data (i.e., 0:00-23:59) (e.g., ${ }^{18-23}$ ). Among them, the coefficient of temporal overlap of activity 
patterns based on the kernel density estimation ${ }^{24,25}$ has been widely used (e.g., ${ }^{5,26-31}$ ). Frey et al. ${ }^{5}$ argued that the kernel density estimation has greatly improved the level of knowledge available from camera-trap data.

Furthermore, recent studies have assessed the influence of effective sample size for the accuracy and the statistical power when estimating the temporal overlap ${ }^{32,33}$. However, measuring the temporal overlap may sometimes be insufficient to assess species interactions correctly, as this method evaluates the overlaps/differences in diel activity patterns between a focal species pair throughout the day, from 0:00 to $23: 59$, from a dataset pooled during a sampled period. For example, if two species are both nocturnal, a subordinate (i.e., smaller) species may coexist with a dominant species by avoiding direct encounters with the larger competitor at fine time scale (e.g., dozens of minutes or several hours), even if the coefficient of temporal overlap shows a high value ${ }^{34}$. Nowadays, the spatio-temporal niche partitioning has been measured to assess behavioral avoidance by focusing on the time-to-encounter between individuals of different species (e.g., ${ }^{34-40}$ ). Indeed, Karanth et al. ${ }^{34}$ found a large overlap in diel activity patterns between tigers (Panthera tigris) and leopards (Panthera pardus), while demonstrating their behavioral avoidance by using the time-to-encounter analysis. Similarly, Paúl et al. ${ }^{39}$ also found a large overlap in diel activity patterns between side-striped jackals (Canis adustus) and African wolves (Canis lupaster), while indicating the occurrence of some behavioral avoidance using the time-to-encounter analysis, with side-striped jackals taking longer than expected to be detected after the occurrence of African wolves. These results suggest that the evaluation of behavioral avoidance at fine time scales using the time-to-encounter analysis may provide an understanding of the mechanisms of species coexistence that cannot be detected by only estimating the temporal overlap. When different analytical approaches detect different niche partitioning results, the mechanisms of species coexistence may be misinterpreted. To avoid this, it is important to evaluate temporal niche partitioning using multiple analytical approaches. Although a previous study evaluated the detection power of the temporal overlap and the time-to-encounter analysis separately ${ }^{33}$, comparative evaluations of multiple analytical methods have not been conducted yet.

To address this methodological issue, we compared multiple analytical methods to accurately evaluate the temporal partitioning among three sympatric mesocarnivores. We used the temporal overlap, temporal co-occurrence analysis, and the time-to-encounter analysis (Fig. 1). Our focal species were red foxes, raccoon dogs (Nyctereutes procyonoides), and Japanese martens (Martes melampus). Red foxes and genus Martes individuals are widespread in the Northern Hemisphere ${ }^{41,42}$, and raccoon dogs are widely distributed in East Asia and Russian Far East and have also been introduced in Europe ${ }^{43}$. In addition, the red foxes and raccoon dogs belong to the same family (i.e., Canidae), and differ in body size from the Japanese martens ${ }^{42}$, indicating their potential competitive interactions, according to Donadio \& Buskirk ${ }^{11}$. Therefore, our focal species are ideal to assess the role of temporal partitioning in mesocarnivores sympatry. In this study, we proposed the temporal co-occurrence analysis (Fig. 1b), adapted from the spatial co-occurrence analysis ${ }^{44,45}$, which is a method used to assess the spatial cooccurrence of multiple species by using presence-absence data (e.g. ${ }^{46-49}$ ). Furthermore, we also 
performed the time-to-encounter analysis (Fig. 1c) based on the concept of multi-response permutation procedures, according to Karanth et al. ${ }^{34}$. We performed this analysis in two ways: using multiple time scales which ranged from several minutes to several days or weeks, according to the previous study 34 , and only using nighttime unit scale to address diel activities of the focal species (for more details see 'Methods').

\section{Methods}

\section{Study area}

We collected animal images by camera-trapping in the Experimental Forest of Yamagata University in Yamagata Prefecture, northeastern Japan, belonging to a cool temperate climatic zone $\left(38^{\circ} 33^{\prime} \mathrm{N}\right.$, $139^{\circ} 51^{\prime} \mathrm{E}$; Fig. 2). The annual mean temperature is approximately $10.5^{\circ} \mathrm{C}$, and the annual precipitation is approximately $3,300 \mathrm{~mm}$ (average from 2019 to 2020). The altitude ranges from approximately 230 to $850 \mathrm{~m}$. The study area has heavy snowfall, with the maximum snow depth exceeding $3 \mathrm{~m}$ in winter. Apart from the three focal mesocarnivores in this study, seven other medium- and large-sized mammals were present in the study area: the Japanese hare (Lepus brachyurus), Japanese macaque (Macaca fuscata), masked palm civet (Paguma larvata), Japanese badger (Meles anakuma), Asiatic black bear (Ursus thibetanus), Japanese weasel (Mustela itatsi), and Japanese serow (Capricornis crispus) ${ }^{50}$. The major canopy species are beeches (Fagus crenata), oaks (Quercus crispula), maples (Aceraceae), and cedar (Cryptomeria japonica) plantations.

\section{Camera trapping}

Between May 1 and October 31, 2019 and 2020, we set 18 camera-trap sites using infrared-triggered cameras (models \#BTC-6HD-940, Browning, AL, USA and \#BTC-6HD-APX, Browning, AL, USA) (Fig. 2; Supplementary Table S1). Previous studies have reported that the effective detection distance of infraredtriggered cameras is shorter with higher vegetation density ${ }^{51}$ and that the detection rate of cameras is higher on forest roads than in forests ${ }^{52}$. To mitigate the change in the detection distance due to the increase in vegetation density, and because the detection rates of the three focal species were also high on the forest road in this study area ${ }^{50}$, we installed all cameras on trees along to a forest road, approximately $1.5 \mathrm{~m}$ above the ground. We programmed the cameras to capture three images for each trigger, with a $1 \mathrm{~min}$ delay between each trigger. We identified the focal species from the three images and recorded the species, camera-trap site, date, and time as a single detection data.

\section{Data analysis}

\section{Spatial autocorrelation}


Positive spatial autocorrelation can arise because of repeated detections of the species and can affect the assessment of species interactions when cameras are set close together within a small area ${ }^{21}$. To assess the positive spatial autocorrelation, we used the Mantel's correlogram ${ }^{53}$ before analyzing the temporal niche partitioning. Correlation indices were calculated using 10,000 randomizing simulations based on the number of detections of each target species per day per camera and the longitudinal/latitudinal positions of cameras. According to Tsunoda et al. ${ }^{21}$, when we collected consecutive detections of the same species at a camera-trap site within $30 \mathrm{~min}$, we treated it as a single sample. This analysis was performed using the 'vegan' package ${ }^{54}$ in statistical software $\mathrm{R}^{55}$ ver. 3.5.2.

\section{Temporal overlap}

We first estimated the diel activity patterns of target species as a probability density function using the kernel density estimation ${ }^{24}$. To determine the interspecific temporal overlap (Fig. 1a), we estimated the coefficient of temporal overlap $(D)$ for each species-pair, which ranged from 0 (no overlap) to 1 (complete overlap) ${ }^{24,25}$. This is defined as the area under the curve that is formed by taking the minimum of the two density functions at each time point ${ }^{56}$. Ridout $\&$ Linkie ${ }^{24}$ developed three estimation methods $\left(D_{1}, D_{4}\right.$, and $D_{5}$ ) for estimating the overlap of diel activity patterns. According to Meredith \& Ridout ${ }^{25}$, we used the $D_{4}$ method because each focal species dataset contained more than 75 samples. We defined the $D \leq 0.5$ as "low", $0.5<D \leq 0.75$ as "moderate", and $D>0.75$ as "high", according to Monterroso et al. ${ }^{19}$. To assess the reliability of the $D$ statistic and its $95 \%$ confidence intervals $(C l)$, we performed a smoothed bootstrap with 10,000 bootstrap samples ${ }^{25}$. To reduce duplicate counts of the same individual, when we collected consecutive detections of the same species at a camera-trap site within $30 \mathrm{~min}$, we treated it as a single sample (e.g., ${ }^{19,21,28,30,57}$ ). These analyses were performed using the 'overlap' package ${ }^{25}$ in R.

\section{Temporal co-occurrence analysis}

To assess whether the three target species avoid each other in units of one night, we performed the temporal co-occurrence analysis using a matrix containing the presence-absence data per night at each camera site for each species pair (Fig. 1b). We only analyzed nighttime because the three target species were nocturnal in this study area ${ }^{30,57}$. We used two methods: the probabilistic co-occurrence analysis ${ }^{45}$ that compares the observed data with simulated data generated by combinatorics, and the checkerboard score $^{44}$ that compares the observed data with simulated data generated through 1,000 randomizations. Both methods determine the probability that the observed frequency of co-occurrence of two species is less than, greater than, or not different from the expected frequency, if the two species occurred independently from each other in units of one night. We defined one night as $60 \mathrm{~min}$ before sunset to 60 min after sunrise. We obtained the sunrise and sunset times for each survey day by using the $\mathrm{R}$ package 'rSetDayNightAttr' ${ }^{58}$. The analyses were performed using the 'cooccur' and 'vegan' packages ${ }^{45,54}$ in R.

\section{Time-to-encounter analysis}

To assess the behavioral avoidance between different species by using the time-to-encounter analysis (Fig. 1c), we used multi-response permutation procedures ${ }^{59}$ (hereafter, MRPP) according to Karanth et 
al. ${ }^{34}$. To determine competitive dominances among the three target species, we considered the before/after occurrence (e.g. red fox (before) - raccoon dog (after), raccoon dog - red fox) for the detected species when calculating the time-to-encounter across each species pair ${ }^{39}$. For this procedure, we created matrix of detection records for each species consisting of the camera-trap site, date, and time. For every detection record, we calculated the minimum time to the subsequent detection between species pairs for each camera-trap site in each survey duration (seven sites: May 1-Oct 31, 2019, four sites: Aug 19-Oct 31, 2019, four sites: May 1-Oct 31, 2020, eight sites: Aug 24-Oct 31, 2020). Thus, for each species pair, we obtained a set of observed times-to-encounter. To compare this to a random expectation (i.e., a null model representative of neither segregation nor aggregation), we randomly permutated the detection records of subsequent species among camera-trap sites with the same survey duration. We used this random permutation to re-calculate the time-to-encounter and repeated this process 1,000 times $^{34}$. Finally, we compared the observed median time-to-encounter and the 1,000 medians of the random permutations. We finally calculated the $p$-values as the proportion of times the observed median was larger than the medians of the random permutations ${ }^{34}$. A large $p$-value indicates spatio-temporal aggregation (i.e., the observed time-to-encounter was shorter than the random expectation) while a small $p$-value indicates spatio-temporal segregation ${ }^{34}$. In the previous studies that have conducted time-toencounter analysis (e.g., ${ }^{34,36}$ ), consecutive detections of the same species less than 1 min apart were collapsed into a single sample. In this study, we set the cameras' interval to $1 \mathrm{~min}$, therefore we used all detection data for this analysis. We used statistical software $\mathrm{R}$ for this analysis.

In the MRPP, the time-to-encounter data between two species may span multiple days ${ }^{34}$. However, our focal species are typically nocturnal ${ }^{30,57}$ and the time-to-encounter data may include daytime when they are inactive or at the resting sites. Considering these factors, we performed the MRPP with an upper limit on the time-to-encounter data to focus on a more detailed scale. To focus on the nocturnal activity time of the target species, we used only the time-to-encounter data within $15 \mathrm{~h}$, and assessed the behavioral avoidance between species using the same calculation method and evaluation criteria in the MRPP described above (hereafter, 15h-MRPP). We defined the time-to-encounter within $15 \mathrm{~h}$ as the longest nighttime during the survey period $(13 \mathrm{~h})$ with one hour before sunrise and after sunset.

\section{Results}

The total number of camera-days at all camera-trap sites was 2,826, excluding the number of days when the angle of the cameras was changed because of bear attacks or when technical issues occurred. We obtained 705, 735, and 289 detection data of red foxes, raccoon dogs, and Japanese martens, respectively.

We found no positive spatial autocorrelation in the first and second proximal distance classes (Supplementary Fig. S1). This result indicated that proximity between cameras did not affect spatial similarities in detected species. Therefore, we showed that the proximal camera-trap placement had little effect on the assessment of species interactions. 


\section{Analysis of temporal overlap}

The $D$ was higher than 0.75 for all species pairs [red fox-raccoon dog was 0.76 (Cl: $0.70-0.80$ ), red foxJapanese marten was 0.82 (Cl: 0.74-0.86), and raccoon dog-Japanese marten was 0.77 (Cl: $0.71-$ 0.83)] (Fig. 3). Therefore, the diel activity patterns were largely overlapped among the species.

\section{Temporal co-occurrence analysis}

The results of the probabilistic co-occurrence analysis showed that the observed frequency of cooccurrence of red foxes and raccoon dogs and of raccoon dogs and Japanese martens was greater than the expected frequency (Table 1). This indicates that these species pairs significantly co-occurred temporally. The observed frequency of co-occurrence of red foxes and Japanese martens was not different from the expected frequency (Table 1).

Table 1

Results of the probabilistic co-occurrence analysis. Observed cooccurrence is the number of observations in which two species cooccur during one night. Expected cooccurrence represents the expected frequency of two species co-occurring during one night. $P_{\text {less }}$ represents the probability that the two species would co-occur at a frequency smaller than the observed frequency, if the two species had occurred randomly (independently). $P_{\text {greater }}$ represents the probability that the two species would co-occur at a frequency greater than the observed frequency, if the two species had occurred randomly (independently). The number of presence of data was 479 for red foxes, 460 for raccoon dogs, and 239 for Japanese martens. Asterisk represents $P<0.05$.

\begin{tabular}{|lllll|}
\hline Species & Observed cooccurrence & Expected cooccurrence & $\boldsymbol{P}_{\text {less }}$ & $\boldsymbol{P}_{\text {greater }}$ \\
\hline Red fox \& Raccoon dog & 96 & 83.0 & 0.962 & $0.049^{*}$ \\
\hline $\begin{array}{l}\text { Red fox \& } \\
\text { Japanese marten }\end{array}$ & 40 & 43.1 & 0.326 & 0.736 \\
$\begin{array}{l}\text { Raccoon dog \& } \\
\text { Japanese marten }\end{array}$ & 55 & 41.4 & & \\
\hline
\end{tabular}

The results of the checkerboard score showed that the observed frequency of co-occurrence of raccoon dogs and Japanese martens was greater than expected frequency (Table 2), indicating that this species pair significantly co-occurred temporally. The observed frequency of co-occurrence of red foxes and raccoon dogs and of red foxes and Japanese martens was not different from the expected frequencies (Table 2). 
Table 2

Results of the checkerboard score. Simulated C-score represents the mean and confidence interval of a distribution generated using a randomization algorithm. The positive SES (standardized effect size) indicates an observed co-occurrence at a rate smaller than that expected by chance (partitioning); a negative effect size indicates an observed co-occurrence at a rate greater than that expected by chance (co-occurrence). The number of presence of data was 479 for red foxes, 460 for raccoon dogs, and 239 for Japanese martens. Asterisk represents $P<0.05$.

\begin{tabular}{|lllll|}
\hline Species & Observed C-score & $\begin{array}{l}\text { Simulated } \\
\text { C-score }\end{array}$ & SES & $P$ \\
\hline Red fox \& Raccoon dog & 0.040 & $0.042(\mathrm{Cl}: 0.039-0.046)$ & -1.698 & 0.102 \\
\hline Red fox \& Japanese marten & 0.025 & $0.024(\mathrm{Cl}: 0.022-0.026)$ & 0.547 & 0.653 \\
\hline Raccoon dog \& Japanese marten & 0.021 & $0.023(\mathrm{Cl}: 0.022-0.025)$ & -2.359 & $0.023^{*}$ \\
\hline
\end{tabular}

\section{Time-to-encounter analysis}

As a result of the MRPP, the $p$-values for all species pairs were greater than 0.92 , indicating their spatiotemporal aggregations (Fig. 4).

The results of the 15h-MRPP showed that the observed median time-to-encounter and the medians of the random permutations did not differ substantially for all species pairs (Fig. 5). Although a strong evidence for spatio-temporal segregations among species was not detected, the $p$-values for the red fox Japanese marten and raccoon dog - Japanese marten pairs were smaller than those of the other pairs, which were closer to the segregation values than aggregation $(p=0.243, p=0.285$, Fig. 5).

\section{Discussion}

The degree of temporal partitioning differed among the compared analytical methods, which use temporal data differently (Table 3). Although the coefficients of temporal overlap $(D)$ indicated large overlaps in diel activities among all species pairs (Fig. 3), the temporal co-occurrence analyses did not detect co-occurrence nor partitioning between red foxes and Japanese martens (Tables 1 and 2). Further, because of the time-to-encounter analysis, while the MRPP showed spatio-temporal aggregations among all species pairs (Fig. 4), the 15h-MRPP detected marginal segregations in the pairs where martens were detected after the other species (Fig. 5). The coefficient temporal overlap evaluates the overlaps/differences in diel activity patterns between a focal species pair throughout the day, from 0:00 to 23:59, from a dataset pooled during a sampled period. The MRPP evaluates time-to-encounter data with multiple temporal scales that from several hours (i.e., within a night) to several days or weeks (i.e., multiple active and inactive periods). These methods potentially overestimated the temporal niche overlaps, by evaluating longer time periods than their "real" active time. In contrast, both the temporal cooccurrence analysis and the 15h-MRPP evaluated only nighttime data that were consistent with our focal species' activity patterns. Our results suggest that only using the coefficient of temporal overlap or the 
MRPP with multiple-day data was insufficient to accurately determine temporal niche partitioning, specifically when both species show similar activity patterns, such as our focal species. Moreover, the 15h-MRPP evaluates the behavioral avoidances using time-to-encounter data that focused on a finer temporal scale (several hours in a night) than those of the other analytical methods, differentiating the results of temporal niche partitioning from the other analytical methods. Our findings indicated that the evaluation of temporal niche partitioning from multiple analytical methods with different temporal-scale data is necessary.

Table 3

Comparison of all analysis results. + represents a result closer to the overlapping side of the temporal niche and - represents a result closer to the partitioning side of the temporal niche.

\begin{tabular}{|c|c|c|c|c|c|c|}
\hline Species & & $\begin{array}{l}\text { Temporal } \\
\text { overlap }\end{array}$ & $\begin{array}{l}\text { Probabilistic co- } \\
\text { occurrence } \\
\text { analysis }\end{array}$ & $\begin{array}{l}\text { Checkerboard } \\
\text { score }\end{array}$ & MRPP & $\begin{array}{l}\text { 15h- } \\
\text { MRPP }\end{array}$ \\
\hline \multirow{2}{*}{$\begin{array}{l}\text { Red fox \& } \\
\text { Raccoon } \\
\text { dog }\end{array}$} & $\begin{array}{l}\text { Red fox- } \\
\text { Raccoon dog }\end{array}$ & + & + & & + & \\
\hline & $\begin{array}{l}\text { Raccoon dog- } \\
\text { Red fox }\end{array}$ & & & & + & \\
\hline \multirow{2}{*}{$\begin{array}{l}\text { Red fox \& } \\
\text { Japanese } \\
\text { marten }\end{array}$} & $\begin{array}{l}\text { Red fox- } \\
\text { Japanese } \\
\text { marten }\end{array}$ & + & & & + & - \\
\hline & $\begin{array}{l}\text { Japanese } \\
\text { marten-Red } \\
\text { fox }\end{array}$ & & & & + & \\
\hline \multirow{2}{*}{$\begin{array}{l}\text { Raccoon } \\
\text { dog \& } \\
\text { Japanese } \\
\text { marten }\end{array}$} & $\begin{array}{l}\text { Raccoon dog- } \\
\text { Japanese } \\
\text { marten }\end{array}$ & + & + & + & + & - \\
\hline & $\begin{array}{l}\text { Japanese } \\
\text { marten- } \\
\text { Raccoon dog }\end{array}$ & & & & + & \\
\hline
\end{tabular}

Although we did not identify which factors were involved, there are several possibilities for the temporal niche overlaps between the target species in this study (Table 3). First, predators typically synchronize their activities with those of their prey, which results in similar activity patterns among predators using the same prey ${ }^{60,61}$. In Japan, the sympatric red foxes, raccoon dogs, and Japanese martens commonly prey on small mammals ${ }^{62}$. Therefore, the activity patterns of our focal species might be similar due to their synchronization with those of their staple prey species. Second, the timing of the activities of multiple species may coincide with each other due to weather conditions. Hendrichsen \& Tyler ${ }^{63}$ indicated that meteorological factors such as temperature, precipitation, and wind can affect rates of heat loss for wild animals, affecting their activity patterns and narrowing the temporal niche partitioning between species $^{64}$. The results of the 15-MRPP indicated the spatio-temporal avoidance by martens against the 
other two species, though the statistical evidence was marginal ( $p=0.243, p=0.285$, Fig. 5). On a more detailed temporal scale, olfactory cues from competitive species provoke behavioral avoidance ${ }^{65}$. Barrull et al. ${ }^{66}$ demonstrated that the stone martens rarely appeared within 60 min of the detection of the red foxes and European badgers (Meles meles), indicating that smaller martens may detect odors from the larger competitors and avoid them temporally. The Japanese marten is the smallest among our focal mesocarnivores ${ }^{42}$. Odors may have different stimulus residual times that can be perceived by animals depending on the type of sources, such as passing animals, urine, or feces. Therefore, it may be important to set a temporal scale that considers the type of odor sources in assessing species interactions. Our results suggest that factors affecting the assessment of species interactions (i.e., the availability of prey species, weather conditions, and odors) may differ depending on the temporal scales. Therefore, it is important to assess the temporal niche partitioning on temporal scales within which species interactions can be detected.

The temporal co-occurrence analyses in units of one night performed in this study did not detect cooccurrence nor partitioning between red foxes and Japanese martens (Tables 1 and 2), even though their diel activities highly overlapped (Fig. 3). This indicated that the overlaps of diel activity patterns were not always consistent to the temporal niche overlaps. This study suggested that the temporal co-occurrence analysis may be an alternative method to assess the temporal interactions between competitive species that cannot be detected by the overlaps of diel activity patterns. The temporal co-occurrence analyses might have methodological advantages, owing to their use of presence-absence data. For example, presence-absence data are typically available in large amounts of data, compared to those of other methods, such as the MRPP. Indeed, Tattersall et al. ${ }^{67}$ indicated that the assessment of the temporal niche partitioning at fine temporal scales required large amounts of the detection data and applied presence-absence data for the assessment. Further, the spatial co-occurrence analysis has been often used in studies focused on spatial niche partitioning and its associated factors (e.g., weather conditions or geo-environmental gradients ${ }^{48}$ ). Therefore, the knowledge and methodology applied in spatial cooccurrence studies would be applicable to temporal assessments.

In this study, we also assessed the before/after occurrence differences in a species pair (e.g., red fox (before) - raccoon dog (after), and raccoon dog - red fox) in the MRPP and 15h-MRPP analyses; the results differed depending on the species-occurrence order (Figs. 4 and 5). However, many previous studies disregarded the effects of this order ${ }^{34,37,38,68}$ (but also see Paúl et al. ${ }^{39}$ ). This study suggested the necessity of the time-to-encounter analysis with the species-occurrence order replacement, if the competitive dominances among focal species are not clear. Although the 15h-MRPP analyses indicated the possibility of behavioral avoidances by Japanese martens to the lager species, there were weak evidences in the statistical assessments (Fig. 5), possibly due to the small sample sizes (Fig. 5). Niedballa et al. ${ }^{33}$ indicated that more than 100 samples per species are needed to perform the time-toencounter analysis with statistical validations. Our findings suggest that the time-to-encounter analysis with a fine temporal scale dataset may be a powerful tool to assess spatio-temporal partitioning between nocturnal carnivores, when the sample sizes of both species are adequate. 
We demonstrated the importance of evaluating the temporal niche partitioning between carnivores on a temporal scale within which species interactions can be detected by using multiple analytical methods with different uses of temporal data. The results of the assessment of the niche partitioning differed depending on the analytical methods, and therefore the choice of analytical approach is important for understanding the mechanisms of species coexistence based on their temporal niche partitioning. Our results suggested that only using the coefficient of temporal overlap to determine the temporal niche partitioning between species is insufficient for assessing species interactions. Therefore, we recommend the use of multiple methods by temporal co-occurrence analysis and/or time-to-encounter analysis in addition to the evaluation by the temporal overlap. Our results also suggest that the factors affecting species interactions may differ depending on the temporal scales. It is important to assess temporal niche partitioning on a detailed temporal scale within which species interactions can be detected by using the time-to-encounter analysis with an upper limit on the time-to-encounter data. Adopting this approach would provide a better understanding of the mechanisms determining species coexistence.

\section{Declarations}

\section{Data availability statement}

The datasets generated during and/or analyzed during the current study are available from the corresponding author on reasonable request.

\section{Acknowledgments}

This study was conducted with the permission of the owner of the forests in the study area. We thank the staff of the Experimental Forest of Yamagata University and the associates of Yamagata University for supporting our field survey.

\section{Author contributions statement}

R.W., H.T., and M.U.S. conceived the study and wrote the main text. R.W. collected data, performed analysis, and prepared tables and figures. All authors reviewed the manuscript.

\section{Additional information}

\section{Competing Interests}

The authors declare no competing interests.

\section{References}

1. Gause, G. F. Experimental analysis of Vito Volterra's mathematical theory of the struggle for existence. Science. 79, 16-17 (1934). 
2. Amarasekare, P. Competitive coexistence in spatially structured environments: A synthesis. Ecol. Lett. 6, 1109-1122 (2003).

3. HilleRisLambers, J., Adler, P. B., Harpole, W. S., Levine, J. M. \& Mayfield, M. M. Rethinking community assembly through the lens of coexistence theory. Annu. Rev. Ecol. Evol. Syst. 43, 227-248 (2012).

4. Wisz, M. S. et al. The role of biotic interactions in shaping distributions and realised assemblages of species: Implications for species distribution modelling. Biol. Rev. 88, 15-30 (2013).

5. Frey, S., Fisher, J. T., Burton, A. C. \& Volpe, J. P. Investigating animal activity patterns and temporal niche partitioning using camera-trap data: challenges and opportunities. Remote Sens. Ecol. Conserv. 3, 123-132 (2017).

6. Davis, C. L. et al. Ecological correlates of the spatial co-occurrence of sympatric mammalian carnivores worldwide. Ecol. Lett. 21, 1401-1412 (2018).

7. Durant, S. M. Competition refuges and coexistence: An example from Serengeti carnivores. J. Anim. Ecol. 67, 370-386 (1998).

8. Fedriani, J. M., Fuller, T. K., Sauvajot, R. M. \& York, E. C. Competition and intraguild predation among three sympatric carnivores. Oecologia 125, 258-270 (2000).

9. Kamler, J. F., Ballard, W. B., Gilliland, R. L. \& Mote, K. Spatial relationships between swift foxes and coyotes in northwestern Texas. Can. J. Zool. 81, 168-172 (2003).

10. Vanak, A. T. et al. Moving to stay in place: Behavioral mechanisms for coexistence of African large carnivores. Ecology 94, 2619-2631 (2013).

11. Donadio, E. \& Buskirk, S. W. Diet, morphology, and interspecific killing in carnivora. Am. Nat. 167, 524-536 (2006).

12. Tsunoda, H. et al. Food niche segregation between sympatric golden jackals and red foxes in central Bulgaria. J. Zool. 303, 64-71 (2017).

13. Palomares, F. \& Caro, T. M. Interspecific killing among mammalian carnivores. Am. Nat. 153, 492508 (1999).

14. Linnell, J. D. C. \& Strand, O. Interference interactions, co-existence and conservation of mammalian carnivores. Divers. Distrib. 6, 169-176 (2000).

15. Kamler, J. F., Stenkewitz, U., Klare, U., Jacobsen, N. F. \& MacDonald, D. W. Resource partitioning among cape foxes, bat-eared foxes, and black-backed jackals in South Africa. J. Wildl. Manage. 76, 1241-1253 (2012).

16. Di Bitetti, M. S., Di Blanco, Y. E., Pereira, J. A., Paviolo, A. \& Pírez, I. J. Time partitioning favors the coexistence of sympatric crab-eating foxes (Cerdocyon thous) and Pampas foxes (Lycalopex gymnocercus). J. Mammal. 90, 479-490 (2009).

17. Lesmeister, D. B., Nielsen, C. K., Schauber, E. M. \& Hellgren, E. C. Spatial and temporal structure of a mesocarnivore guild in midwestern north America. Wildl. Monogr. 191, 1-61 (2015).

18. Di Bitetti, M. S., De Angelo, C. D., Di Blanco, Y. E. \& Paviolo, A. Niche partitioning and species coexistence in a Neotropical felid assemblage. Acta Oecologica 36, 403-412 (2010). 
19. Monterroso, P., Alves, P. C. \& Ferreras, P. Plasticity in circadian activity patterns of mesocarnivores in Southwestern Europe: Implications for species coexistence. Behav. Ecol. Sociobiol. 68, 1403-1417 (2014).

20. Tsunoda, H., Ito, K., Peeva, S., Raichev, E., \& Kaneko, Y. Spatial and temporal separation between the golden jackal and three sympatric carnivores in a human-modified landscape in central Bulgaria. Zool. Ecol. 28, 172-179 (2018).

21. Tsunoda, H. et al. Spatio-temporal partitioning facilitates mesocarnivore sympatry in the Stara Planina Mountains, Bulgaria. Zoology 141, 125801 (2020).

22. Ramesh, T., Kalle, R., Sankar, K. \& Qureshi, Q. Spatio-temporal partitioning among large carnivores in relation to major prey species in Western Ghats. J. Zool. 287, 269-275 (2012).

23. Gómez-Ortiz, Y., Monroy-Vilchis, O. \& Castro-Arellano, I. Temporal coexistence in a carnivore assemblage from central Mexico: temporal-domain dependence. Mammal Res. 64, 333-342 (2019).

24. Ridout, M. S. \& Linkie, M. Estimating overlap of daily activity patterns from camera trap data. J. Agric. Biol. Environ. Stat. 14, 322-337 (2009).

25. Meredith, M. \& Ridout, M. Overlap: Estimates of coefficient of overlapping for animal activity patterns. Package 'overlap'https://cran.r-project.org/web/packages/overlap/index.html (2018).

26. Marinho, P. H., Fonseca, C. R., Sarmento, P., Fonseca, C. \& Venticinque, E. M. Temporal niche overlap among mesocarnivores in a Caatinga dry forest. Eur. J. Wildl. Res. 66, 1-13 (2020).

27. Vilella, M., Ferrandiz-Rovira, M. \& Sayol, F. Coexistence of predators in time: Effects of season and prey availability on species activity within a Mediterranean carnivore guild. Ecol. Evol. 10, 1140811422 (2020).

28. Zhao, G. et al. Spatio-temporal coexistence of sympatric mesocarnivores with a single apex carnivore in a fine-scale landscape. Glob. Ecol. Conserv. 21, e00897 (2020).

29. Farmer, M. J., Allen, M. L., Olson, E. R., Van Stappen, J. \& Van Deelen, T. R. Agonistic interactions and island biogeography as drivers of carnivore spatial and temporal activity at multiple scales. Can. J. Zool. 99, 309-317 (2021).

30. Watabe, R. \& Saito, M. U. Diel Activity Patterns of Three Sympatric Medium-Sized Carnivores during Winter and Spring in a Heavy Snowfall Area in Northeastern Japan. Mammal Study 46, 69-75 (2021).

31. Viviano, A. et al. Spatiotemporal Overlap between the European Brown Hare and Its Potential Predators and Competitors. Animals 11, 562 (2021).

32. Lashley, M. A. et al. Estimating wildlife activity curves: comparison of methods and sample size. Sci. Rep. 8, 4173 (2018).

33. Niedballa, J., Wilting, A., Sollmann, R., Hofer, H. \& Courtiol, A. Assessing analytical methods for detecting spatiotemporal interactions between species from camera trapping data. Remote Sens. Ecol. Conserv. 5, 272-285 (2019). 
34. Karanth, K. U. et al. Spatio-temporal interactions facilitate large carnivore sympatry across a resource gradient. Proc. R. Soc. B Biol. Sci. 284, 20161860 (2017).

35. Cusack, J. J. et al. Revealing kleptoparasitic and predatory tendencies in an African mammal community using camera traps: a comparison of spatiotemporal approaches. Oikos 126, 812-822 (2017).

36. Balme, G. et al. Big cats at large: Density, structure, and spatio-temporal patterns of a leopard population free of anthropogenic mortality. Popul. Ecol. 61, 256-267 (2019).

37. Li, Z. et al. Coexistence of two sympatric flagship carnivores in the human-dominated forest landscapes of Northeast Asia. Landsc. Ecol. 34, 291-305 (2019).

38. Lahkar, D., Ahmed, M. F., Begum, R. H., Das, S. K. \& Harihar, A. Inferring patterns of sympatry among large carnivores in Manas National Park - a prey-rich habitat influenced by anthropogenic disturbances. Anim. Conserv. (2020) doi:10.1111/acv.12662.

39. Paúl, M. J., Layna, J. F., Monterroso, P. \& Álvares, F. Resource Partitioning of Sympatric African Wolves (Canis lupaster) and Side-Striped Jackals (Canis adustus) in an Arid Environment from West Africa. Diversity 12, 477 (2020).

40. Prat-Guitart, M., Onorato, D. P., Hines, J. E. \& Oli, M. K. Spatiotemporal pattern of interactions between an apex predator and sympatric species. J. Mammal. 101, 1279-1288 (2020).

41. Proulx, G. et al. World distribution and status of the genus Martes in 2000. Martens and Fishers (Martes) in Human-Altered Environments 21-76 (Springer, 2005). doi:10.1007/b99487.

42. Ohdachi, S., Ishibashi, Y., Iwasa, M., Fukuki, D. and Saitoh, T. The wild mammals of Japan, 2nd edn. 222-225, 258-259 (Shokadoh Book Seller, Kyoto, 2015).

43. Kauhala, K. and Saeki, M. Nyctereutes procyonoides. The IUCN Red List of Threatened Species. https://www.iucnredlist.org/species/14925/85658776 (2016).

44. Stone, L. \& Roberts, A. The checkerboard score and species distributions. Oecologia $\mathbf{8 5}, \mathbf{7 4 - 7 9}$ (1990).

45. Griffith, D. M., Veech, J. A. \& Marsh, C. J. Cooccur: Probabilistic species co-occurrence analysis in R. J. Stat. Softw. 69, 1-17 (2016).

46. Noor, A., Mir, Z. R., Veeraswami, G. G. \& Habib, B. Activity patterns and spatial co-occurrence of sympatric mammals in the moist temperate forest of the Kashmir Himalaya, India. Folia Zool. 66, 231-241 (2017).

47. de Satgé, J., Teichman, K. \& Cristescu, B. Competition and coexistence in a small carnivore guild. Oecologia 184, 873-884 (2017).

48. Kass, J. M., Tingley, M. W., Tetsuya, T. \& Koike, F. Co-occurrence of invasive and native carnivorans affects occupancy patterns across environmental gradients. Biol. Invasions 22, 2251-2266 (2020).

49. Louppe, V., Herrel, A., Pisanu, B., Grouard, S. \& Veron, G. Assessing occupancy and activity of two invasive carnivores in two Caribbean islands: implications for insular ecosystems. J. Zool. 313, 182194 (2020). 
50. Watabe, R., Saito, M. U., Enari, H. S. \& Enari, H. Mammalian fauna of the Kaminagawa Experimental Forest of Yamagata University detected by camera traps. Tohoku J. For. Sci. 25, 37-40 (2020).

51. Hofmeester, T. R., Rowcliffe, J. M. \& Jansen, P. A. A simple method for estimating the effective detection distance of camera traps. Remote Sens. Ecol. Conserv. 3, 81-89 (2017).

52. Di Bitetti, M. S., Paviolo, A. \& De Angelo, C. Camera trap photographic rates on roads vs. off roads: Location does matter. Mastozoología Neotrop. 21, 37-46 (2014).

53. Borcard, D. \& Legendre, P. Is the Mantel correlogram powerful enough to be useful in ecological analysis? A simulation study. Ecology 93, 1473-1481 (2012).

54. Oksanen, J. et al. Vegan: community Ecology Package. Community ecology package https://cran.rproject.org/web/packages/vegan/index.html (2019).

55. R Core Team. R: A Language Environment for Statistical Computing. R Foundation for Statistical Computing, Vienna, Austria. https://www.r-project.org/ (2018).

56. Linkie, M. \& Ridout, M. S. Assessing tiger-prey interactions in Sumatran rainforests. J. Zool. 284, 224-229 (2011).

57. Watabe, R. \& Saito, M. U. Effects of vehicle-passing frequency on forest roads on the activity patterns of carnivores. Landsc. Ecol. Eng. 17, 225-231 (2021).

58. Furukawa, G. genkiFurukawa/rSetDayNightAttr documentation. https://rdrr.io/github/genkiFurukawa/rSetDayNightAttr/ (2019).

59. Mielke, P. W., Berry, K. J. \& Johnson, E. S. Multi-response permutation proccedures for a priori classifications. Commun. Stat. - Theory Methods 5, 1409-1424 (1976).

60. Kronfeld-Schor, N. \& Dayan, T. Partitioning of Time as an Ecological Resource. Annu. Rev. Ecol. Evol. Syst. 34, 153-181 (2003).

61. Monterroso, P., Alves, P. C. \& Ferreras, P. Catch Me If You Can: Diel Activity Patterns of Mammalian Prey and Predators. Ethology 119, 1044-1056 (2013).

62. Yamamoto, Y. Comparative analyses on food habits of Japanese marten, red fox, badger and raccoon dog in the Mt. Nyugasa, Nagano Prefecture, Japan. Nat. Environ. Sci. Res. 7, 45- 52 (1994).

63. Hendrichsen, D. K. \& Tyler, N. J. C. How the timing of weather events influences early development in a large mammal. Ecology 95, 1737-1745 (2014).

64. Herfindal, I. et al. Weather affects temporal niche partitioning between moose and livestock. Wildlife Biol. (2017) doi:10.2981/wlb.00275.

65. Haswell, P. M., Jones, K. A., Kusak, J. \& Hayward, M. W. Fear, foraging and olfaction: how mesopredators avoid costly interactions with apex predators. Oecologia 187, 573-583 (2018).

66. Barrull, J. et al. Factors and mechanisms that explain coexistence in a Mediterranean carnivore assemblage: An integrated study based on camera trapping and diet. Mamm. Biol. 79, 123-131 (2014).

67. Tattersall, E. R., Burgar, J. M., Fisher, J. T. \& Burton, A. C. Boreal predator co-occurrences reveal shared use of seismic lines in a working landscape. Ecol. Evol. 10, 1678-1691 (2020). 
68. Moll, R. J. et al. Humans and urban development mediate the sympatry of competing carnivores. Urban Ecosyst. 21, 765-778 (2018).

Figures

a) Temporal overlap

- Coefficient of temporal overlap

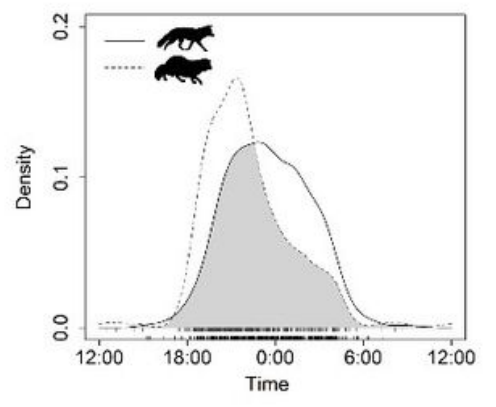

Overlap of diel activity patterns b) Temporal co-occurrence analysis

- Probabilistic co-occurrence analysis

- Checkerboard score

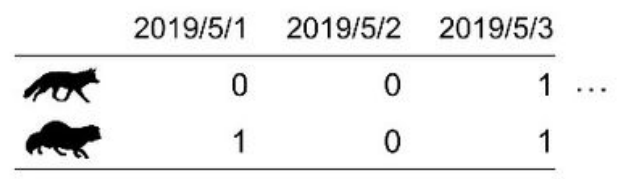

Co-occurrence / partitioning in units of one night c) Time-to-encounter analysis

- MRPP

- 15h-MRPP

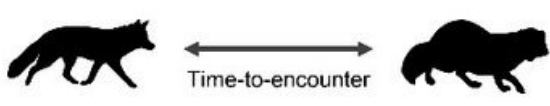

Assessment of behavioral avoidance based on time-to-encounter data

\section{Figure 1}

Images illustrating the different analytical approaches used in this study. 


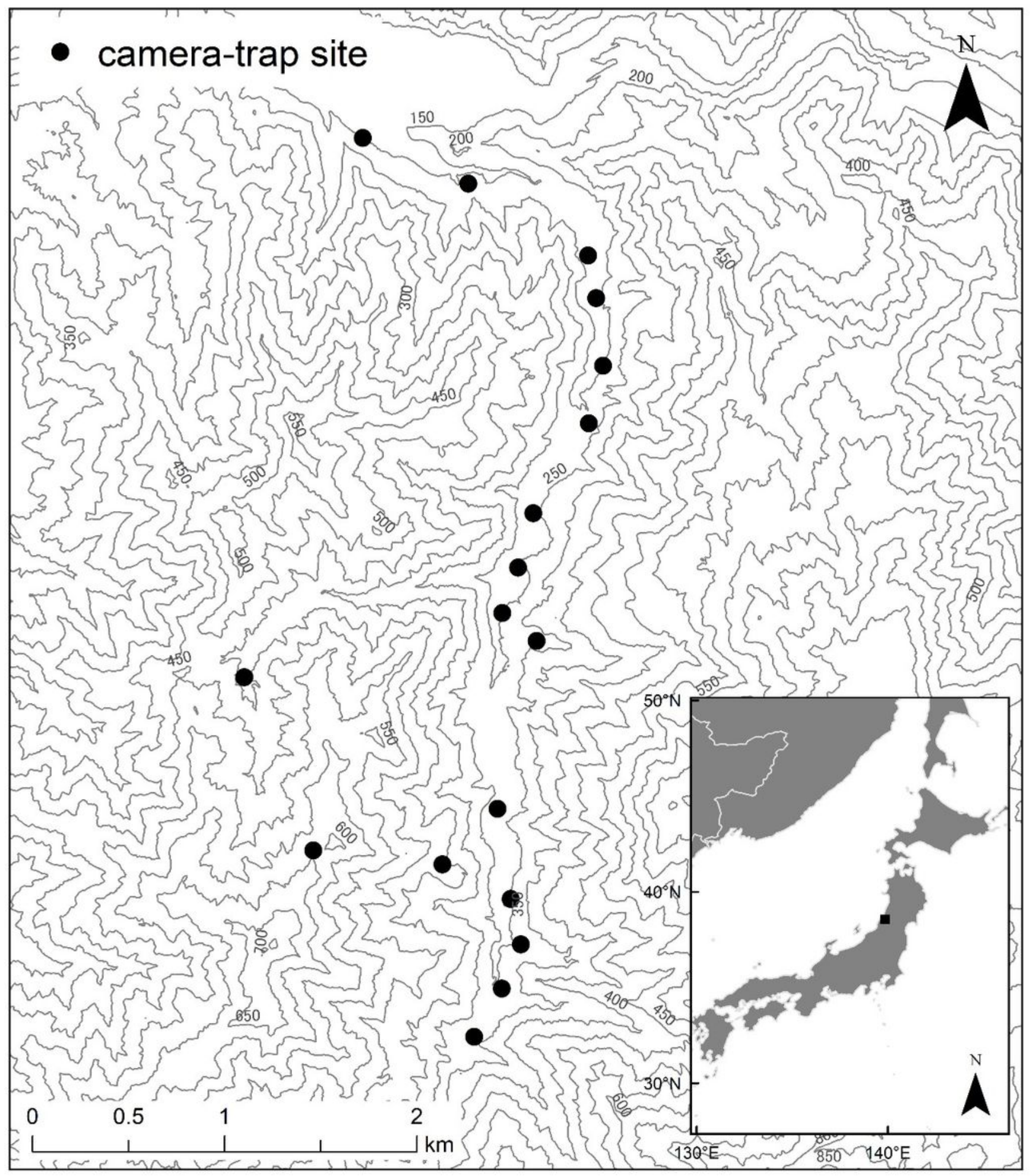

\section{Figure 2}

Study area and locations of camera-trap sites. 

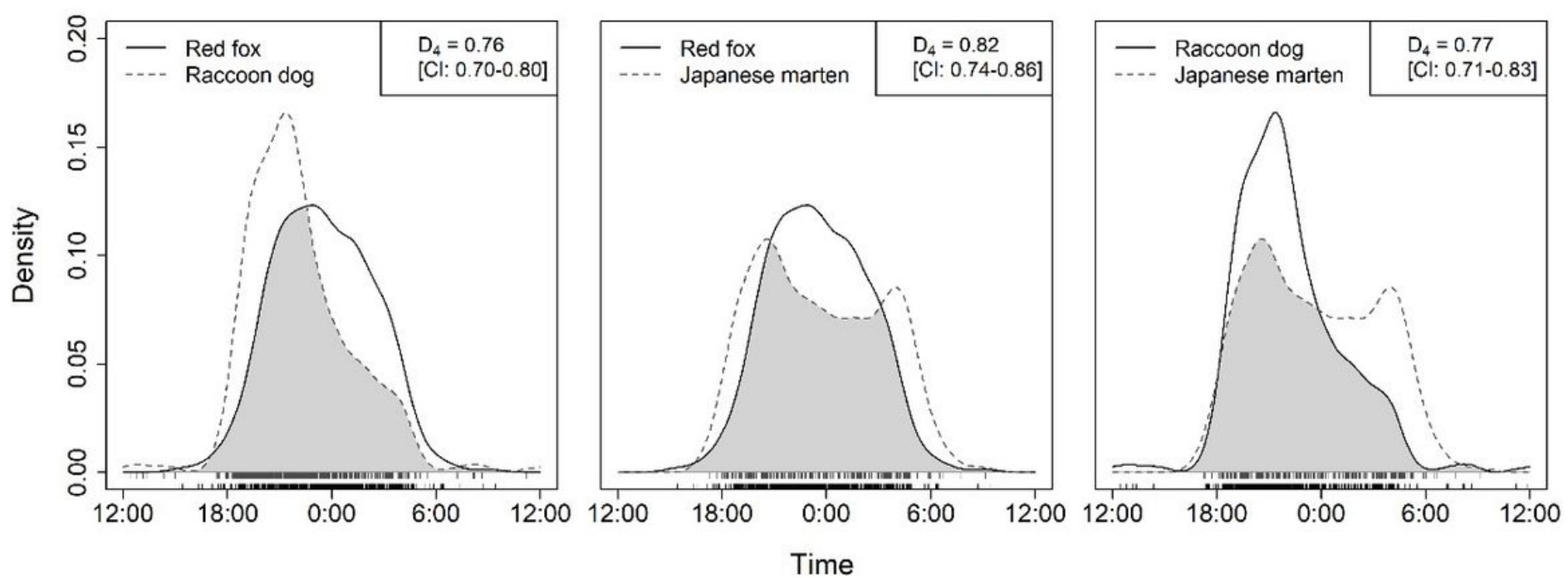

Figure 3

Temporal overlap between species. The gray area indicates the overlap between diel activity patterns. The rug at the bottom of the plot indicates the original observations of activity time. The number of samples used for the kernel density estimation was 669 for red foxes, 691 for raccoon dogs, and 282 for Japanese martens. 

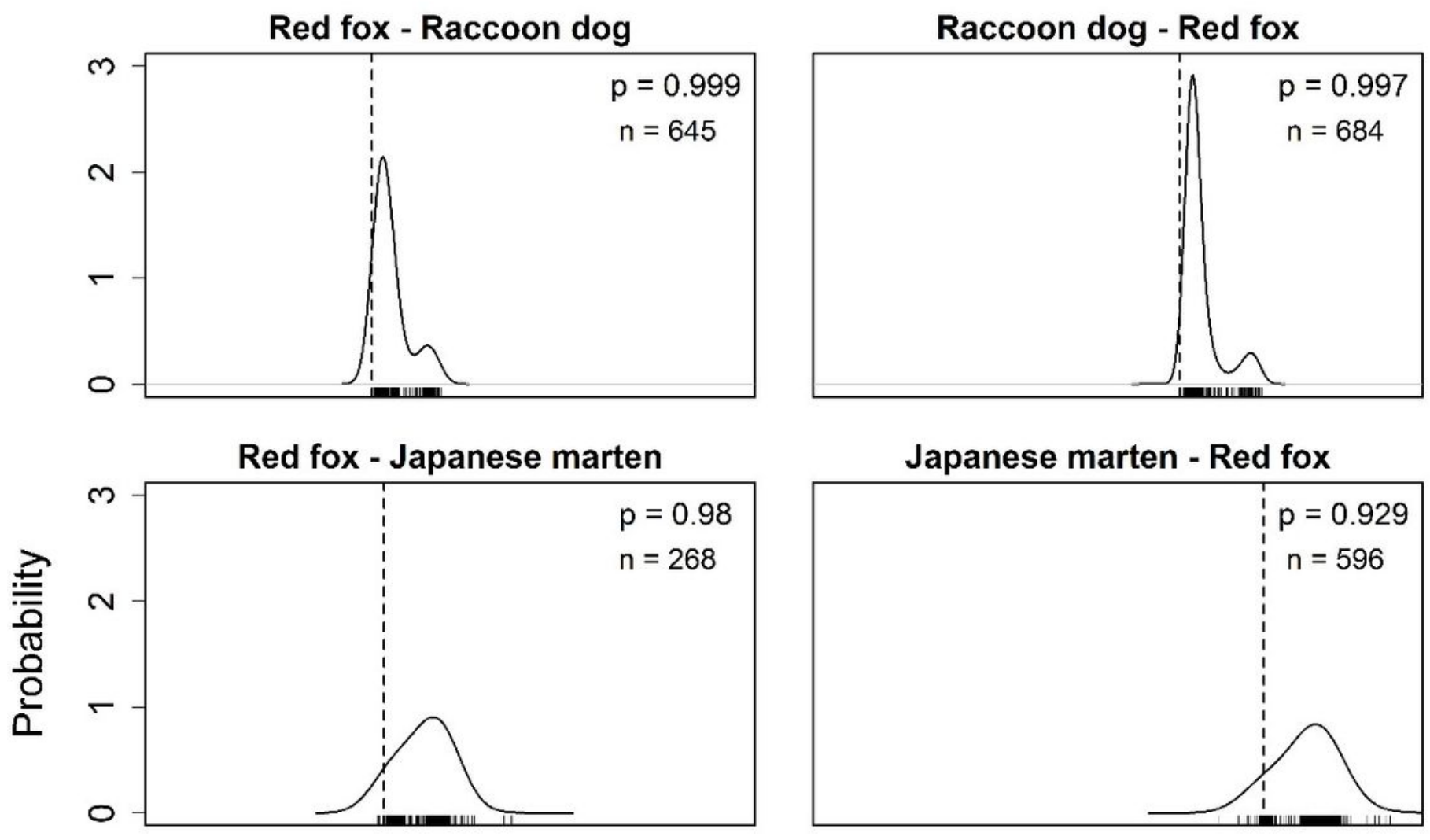

Japanese marten - Red fox
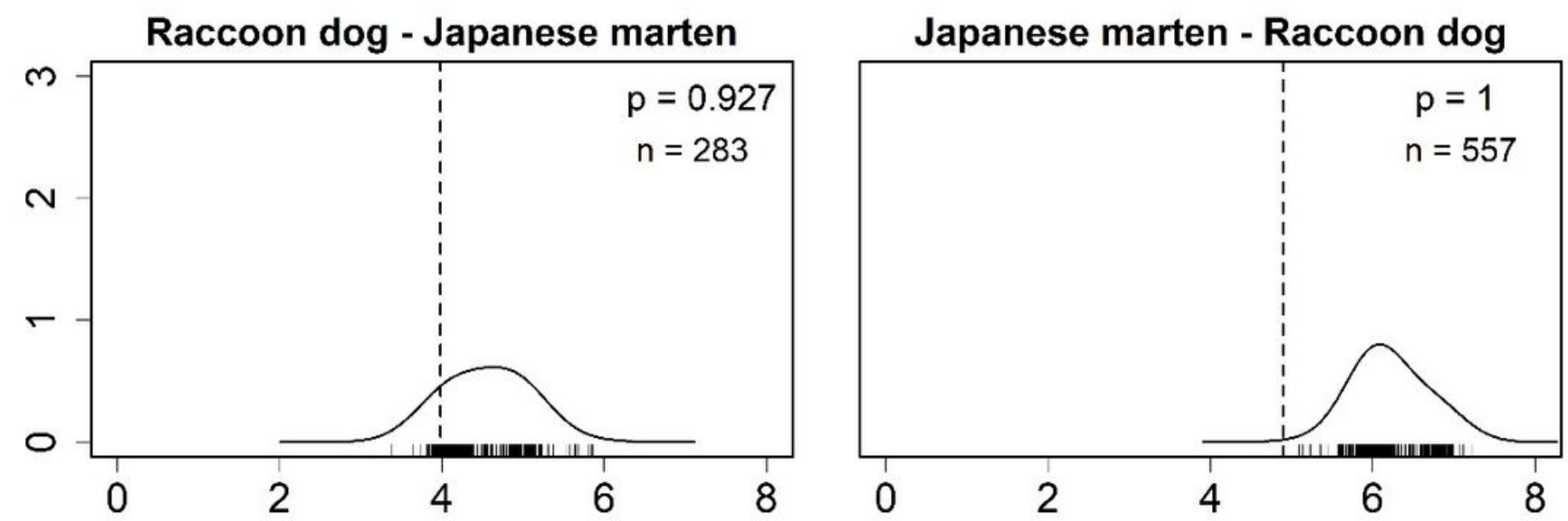

Days

\section{Figure 4}

Results of the time-to-encounter analysis using the MRPP. Red fox-raccoon dog represents that raccoon dogs was detected after red foxes, for example. The dashed vertical lines represent the observed median time-to-encounter between two species. The rug plot represents the 1,000 medians of the random permutations and the curve represents the distribution estimated from them; $\mathrm{n}$ represents the number of the observed time-to-encounter. The p-values indicate the proportion of times the observed median was larger than the medians of the random permutations. 

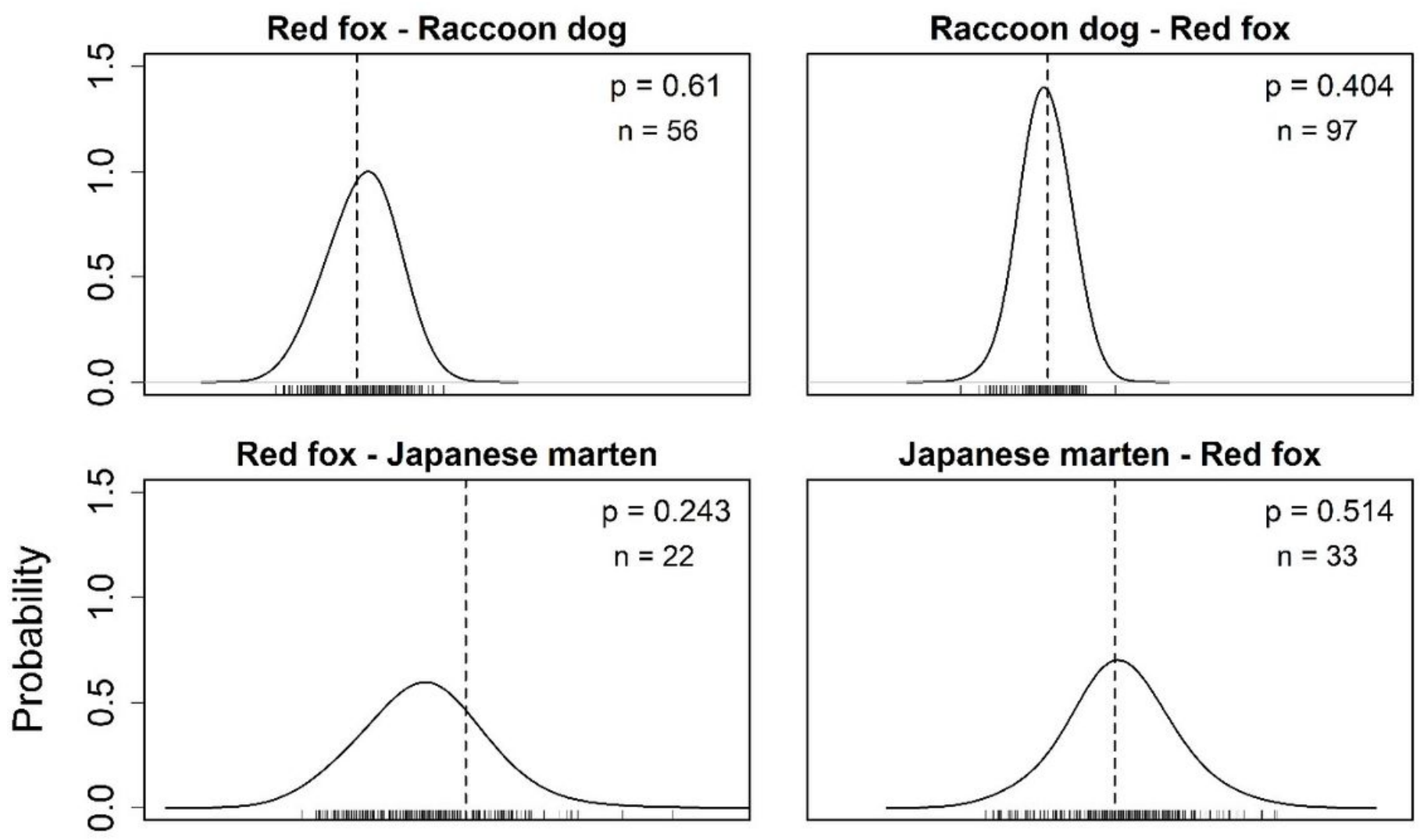

Japanese marten - Red fox
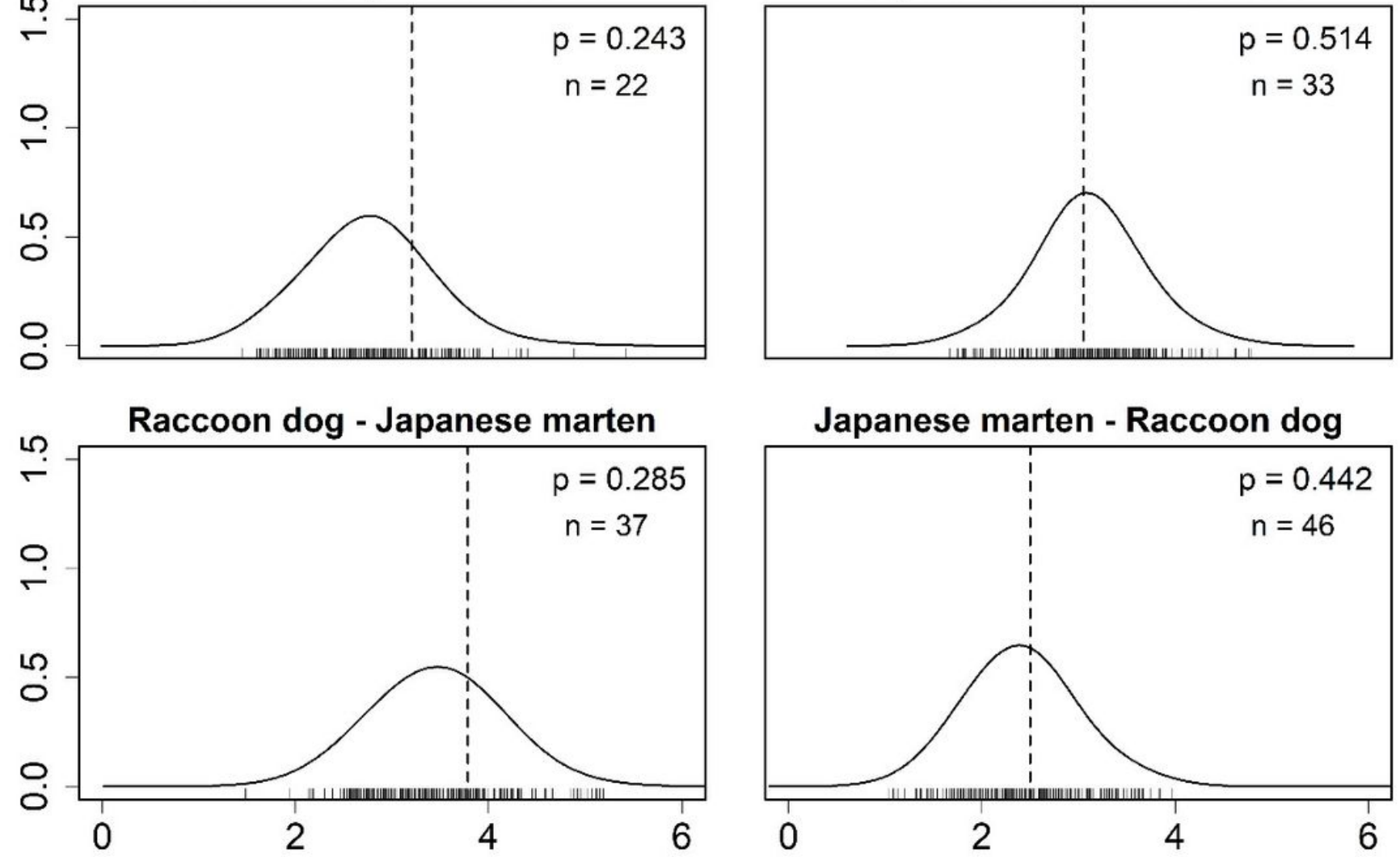

Hours

Figure 5

Results of the time-to-encounter analysis using the 15-MRPP. Red fox-raccoon dog represents that raccoon dogs was detected after red foxes, for example. The dashed vertical lines represent the observed median time-to-encounter between two species. The rug plot represents the 1,000 medians of the random permutations and the curve represents the distribution estimated from them; $\mathrm{n}$ represents the number of the observed time-to-encounter. The p-values indicate the proportion of times the observed median was larger than the medians of the random permutations. 


\section{Supplementary Files}

This is a list of supplementary files associated with this preprint. Click to download.

- SupplementaryinformationWatabeetal.pdf 